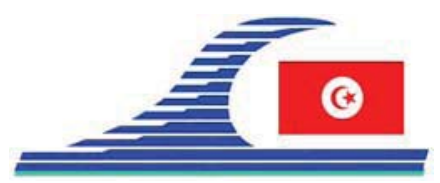

Conférence Méditerranéenne Côtière et Maritime EDITION 1, HAMMAMET, TUNISIE (2009)

Coastal and Maritime Mediterranean Conference

Disponible en ligne - http://www.paralia.fr-Available online

\title{
Atlas numérique des zones inondables par submersion marine du littoral du Languedoc-Roussillon
}

\section{Christophe ESPOSITO ${ }^{1}$}

1. CETE Méditerranée - DREC / SH,

CS 70499, 13593 Aix-en-Provence Cedex 3, France.

christophe.esposito@developpement-durable.gouv.fr

\section{Résumé :}

Le Service du Littoral et des Étangs de la Direction Régionale de l'Équipement du Languedoc Roussillon a confié au CETE Méditerranée l'étude d'un atlas numérique des zones inondables par submersion marine du littoral sableux du Languedoc Roussillon. Une première phase de l'étude a permis de mettre au point une méthodologie basée sur l'utilisation de données informatiques disponibles de nature géomorphologique, historique et topographique. L'atlas numérique permet de produire une cartographie géomorphologique des plaines littorales au contact des plaines alluviales et des formes encaissantes et une cartographie historique des zones inondées. L'article qui suit concerne les départements des Pyrénées-Orientales, de l'Aude de l'Hérault et du Gard.

\section{Mots-clés :}

Tempête - Submersion marine - Atlas des zones inondables - Languedoc-Roussillon

\section{Introduction}

L'objectif de l'article est de présenter une méthodologie de cartographie des zones inondables par submersion marine mise en place, dans un premier temps, sur des secteurs tests (figure 1). Ensuite cette méthodologie a été appliquée sur l'ensemble du littoral du Languedoc Roussillon c'est-à-dire sur les quatre départements que sont les Pyrénées-Orientales, l'Aude, l'Hérault et le Gard.

L'étude méthodologique a été réalisée en deux phases successives sur quatre secteurs tests. Premièrement, nous avons réalisé une phase d'analyse qui se basait sur plusieurs descriptions avec la géomorphologie du littoral, les aménagements réalisés, l'approche historique et une étude topographique. Ensuite, la phase de synthèse a conduit, par le croisement des résultats de l'interprétation géomorphologique, topographique et historique, à l'évaluation et à la cartographie du degré de submersion. 


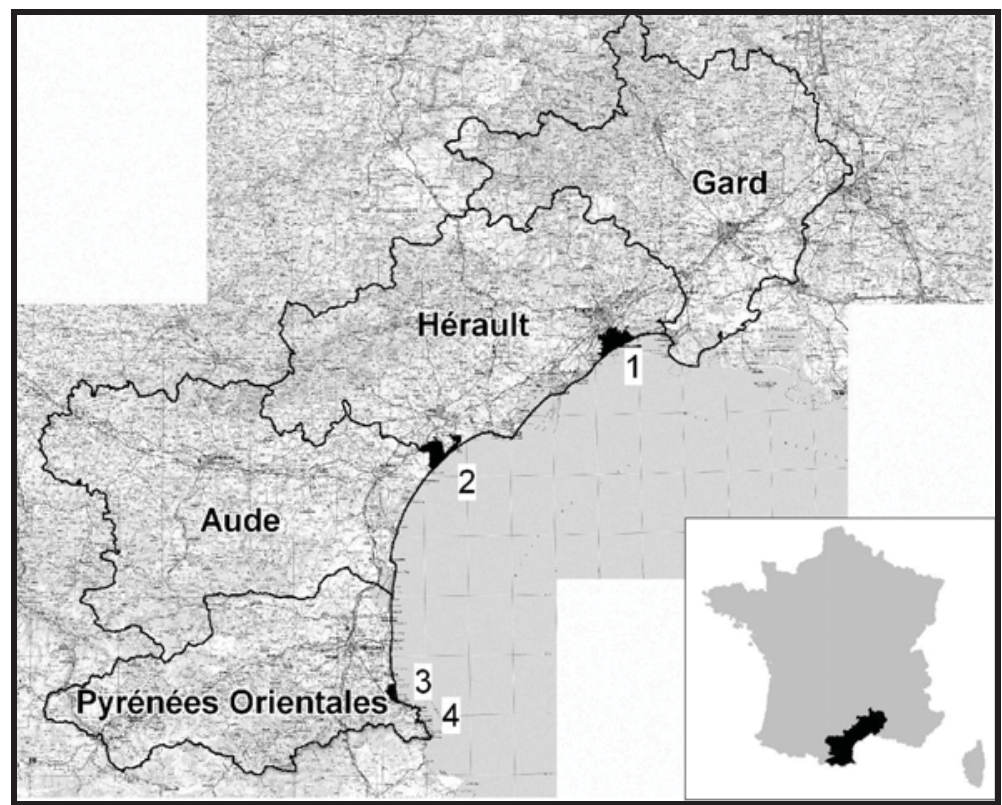

Figure 1. Localisation du secteur d'étude. 1- Palavas-les-flots, 2 - Valras-plage, 3 Argelès-sur-mer, 4 - Banyuls-sur-mer.

\section{Débordement de cours d'eau et submersion marine}

L'approche hydrogéomorphologique permet de déterminer les zones inondables par débordement des cours pour les crues rares à exceptionnelles. Dans le cadre des tempêtes, nous avons essayé de caractériser les différentes entités jouant un rôle dans les submersions marines. Il a été nécessaire de définir chacune d'elles avec la zone marine, la plage vive, les cordons littoraux, la plaine littorale et la plaine alluviale. Nous avons développé une méthode basée sur la géomorphologie du littoral (figure 2). L'objectif est d'identifier des formes et formations caractéristiques du milieu littoral (en contact avec la plaine alluviale) permettant de raccorder un type d'aléa «submersion marine qualitatif $»$.

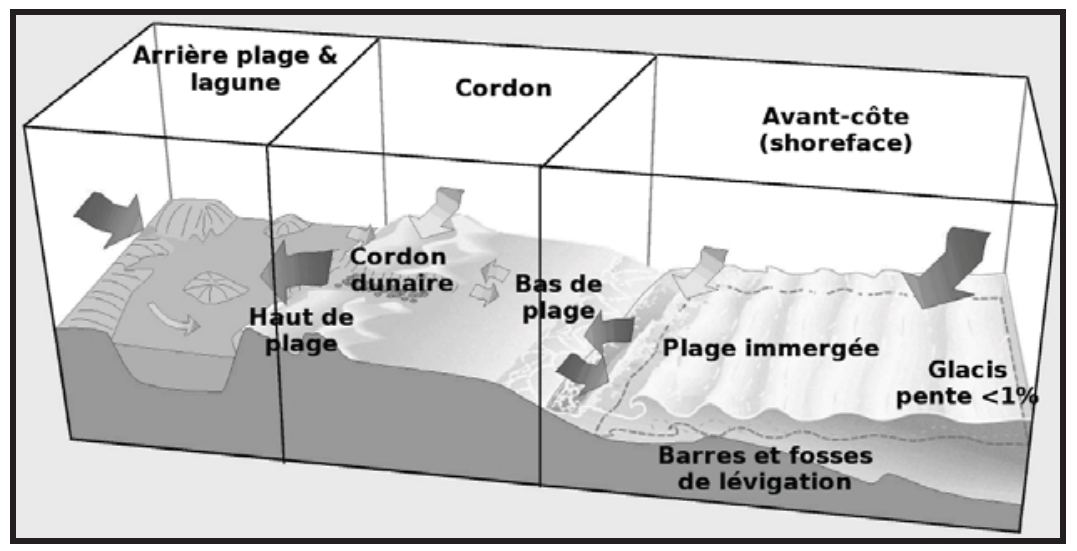

Figure 2. Morphologie d'ensemble du littoral. 


\section{Contenu de l'atlas numérique}

La cartographie géomorphologique est basée sur l'identification d'unités spatiales homogènes. Pour les unités actives du littoral qui sont influencées par un hydrodynamisme marin ou/et fluvial, nous prendrons en considération la plaine alluviale, le cordon littoral et la plaine littorale. Autour de ces unités marquées par une présence d'eau, nous avons identifié les unités encaissantes sans rôle hydrodynamique.

Toutes ces unités sont identifiées et traduites intégralement sous forme de données numériques au format Mapinfo 7.8 appelées «Table». Chaque « Table» est décrite avec son libellé (tableau 1), sa définition, son processus de production, ses caractéristiques informatiques et ses attributs supportés.

Tableau 1. Synthèse de données relatives à cette revue.

\begin{tabular}{|c|c|}
\hline Libellé & Code de la "Table» \\
\hline Zone d'étude & $S_{-} E T U D$ \\
\hline Zone marine & $S \_M A R S$ \\
\hline Réseau hydrographique surfacique & $S \_H Y D R$ \\
\hline Plaine littorale et alluviale & $S \_P L A I$ \\
\hline Cordon littoral & $S \_C O R D$ \\
\hline Encaissant & $S \_E N C S$ \\
\hline Aménagements et ouvrages hydrauliques surfaciques & $S \_O A N T$ \\
\hline Réseau hydrographique linéaire & $L \_H Y D R$ \\
\hline Crête de dune & L_CORD \\
\hline Limite encaissant & L_ENCS \\
\hline Infrastructures et ouvrages hydrauliques linéaires & $L_{-} O A N T$ \\
\hline Aménagement hydrographique linéaire & $L_{-} A M H Y$ \\
\hline Élément d'occupation du sol & $P_{-} O B S$ \\
\hline
\end{tabular}

\section{Cartographie de l'aléa}

C'est à partir des cartes géomorphologiques que nous avons pu éditer des cartes de l'aléa «submersion marine ». A partir des tables informatiques du tableau 1, nous avons pu mettre en place un zonage qui fait apparaître un aléa « qualitatif». C'est seulement à partir de l'interprétation des unités géomorphologiques et une analyse «à dire d'expert » que les cartes d'aléas sur chaque département du Languedoc-Roussillon ont été réalisées. L'aléa est nommé « qualitatif» car, avec cette méthode, nous ne pouvons pas définir de hauteur ou de vitesse de submersion. La figure 3 propose un extrait de cette cartographie. 


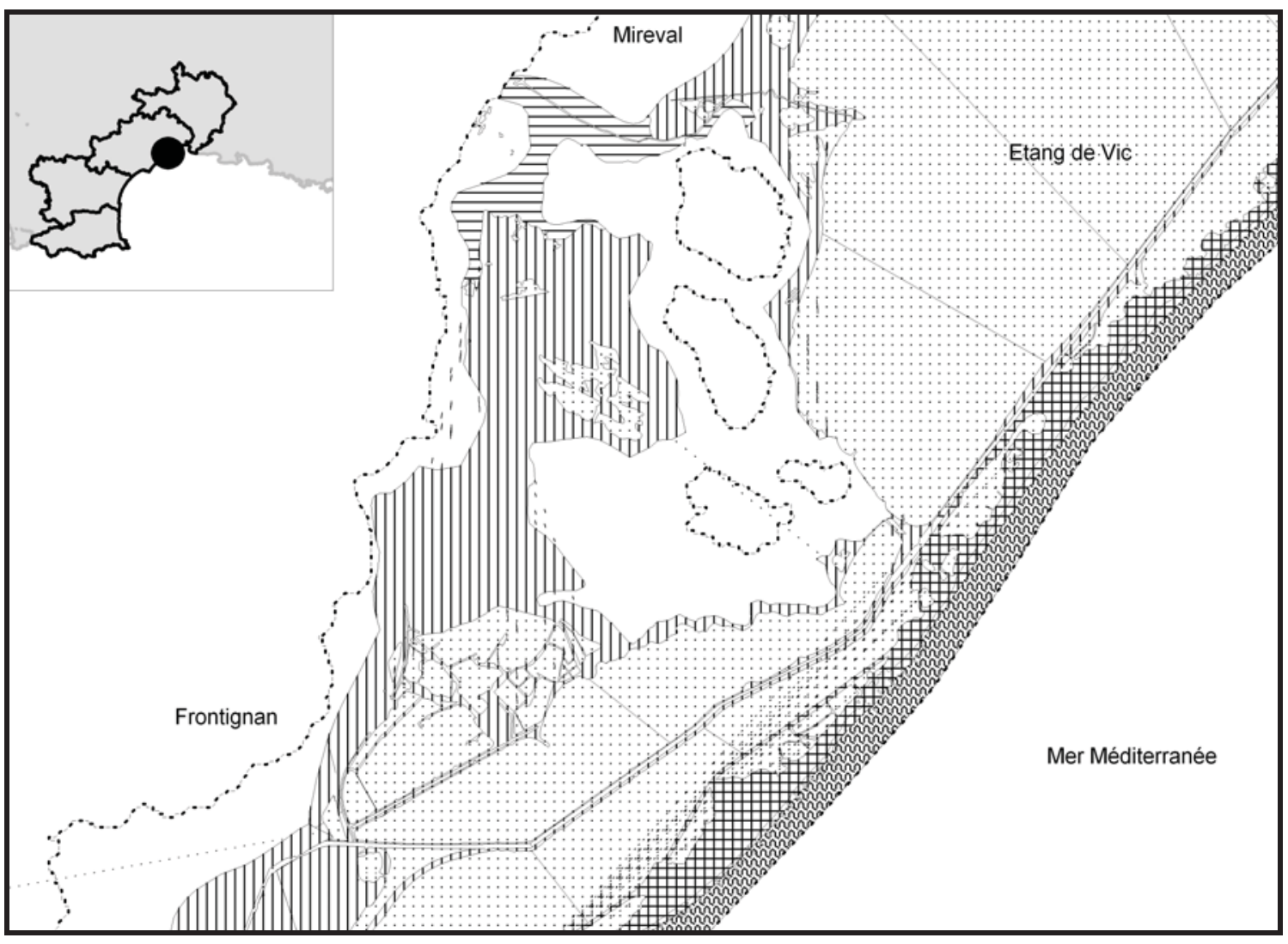

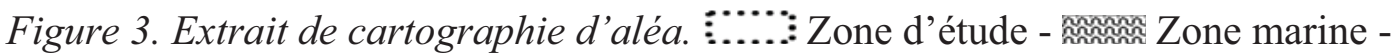
Zone hydrographique - $\square$ Aléa nul - $\rightleftarrows$ Aléa faible - Ш Aléa moyen 坥 Aléa Fort

\section{Discussion et perspectives}

Cette approche naturaliste innovatrice est fondée sur une méthodologie mise au point dans la région du Languedoc-Roussillon. Cette méthodologie a été développée sur plus de $200 \mathrm{~km}$ de littoral sableux et rocheux sur quatre départements. La façade méditerranéenne est caractérisée par des phénomènes de marées faibles (régime microtidal). L'objectif de cette méthodologie est d'être étendue sur d'autres espaces littoraux afin d'observer sa pertinence par rapport au régime des marées (régimes méso-tidaux ou macro-tidaux). Dans les secteurs à plus fortes marrées qu'en Languedoc Roussillon, les tempêtes conjuguées avec une marée de "vive-eau" rend possible la pénétration des terres par les eaux marines. Plus le territoire a des marées importantes, plus l'étendue de la submersion marine évolue temporellement en fonction du cycle des marées. De nouvelles expérimentations en milieu océanique permettront de faire évoluer cette approche et de pouvoir caractériser l'aléa «submersion marine» sur d'importants linéaires de côtes. 